\title{
Incidents de radioprotection signalés à l'ex-OPRI en 1998 et 1999
}

\author{
C. CHALlETON-DE VATHAIRE ${ }^{1}$, P. BOISSON ${ }^{2}$, E. DUBUQUOY ${ }^{3}$, \\ M. BOURGUIGNON ${ }^{4}$, M. VALERO ${ }^{4}$, A. BIAU ${ }^{5}$
}

(Manuscrit reçu le $1^{e r}$ octobre 2002, accepté le 22 mai 2004)

RÉSUMÉ $\quad$ En 1998 et 1999, 160 incidents susceptibles d'avoir entraîné une exposition externe ou interne de travailleurs ou de membres du public et ayant fait l'objet de mesures et/ou d'estimations individuelles ont été signalés à l'ex-OPRI, organisme dont une partie a fusionné avec l'Institut de protection et de sûreté nucléaire (IPSN) en $\mathbf{2 0 0 2}$ pour former l'IRSN. Cette étude décrit les caractéristiques de ces incidents en termes de lieu de survenue, circonstances de découverte et conséquences prévisibles ou réelles. $L$ 'analyse statistique de ces évènements montre une grande diversité des lieux de survenue, les principaux secteurs étant l'industrie nucléaire (57\% des incidents), les laboratoires de recherche (19\%) et le secteur médical (14\%). Au total, 369 personnes ont été concernées par ces 160 incidents et parmi celles-ci, 305 pour des suspicions de contamination, 54 pour celles d'irradiation et enfin 10 pour une suspicion de contamination et d'irradiation concomitantes. Les mesures réalisées ont confirmé la contamination chez la moitié des personnes concernées. En ce qui concerne le risque d'irradiation, sur 64 personnes susceptibles d'avoir été exposées, 34 ont reçu une dose non nulle, supérieure à $50 \mathrm{mSv}$ pour quatre d'entre elles. Compte tenu de la fréquence des incidents de contamination, il serait souhaitable de développer des études spécifiques sur les radionucléides concernés, en particulier ceux utilisés dans les laboratoires de recherche et dans le domaine médical afin d'améliorer l'expertise en cas d'incident dans ces secteurs.

ABSTRACT Radiation protection incidents concerning individuals reported to OPRI between 1998 and 1999.

Between 1998 and 1999, 160 incidents with possible consequences in terms of external or internal exposure of workers or members of the public and with recorded estimation and/or measure of exposure were reported to the former French public radiation protection institute "Office de protection contre les rayonnements ionisants". This study describes the characteristics of these incidents in terms of place and circumstances of discovery and possible or confirmed consequences. There was a broad diversity in the place of discovery, $57 \%$ in nuclear industry, $19 \%$ in research laboratories and $14 \%$ in medical facilities. As a whole, 369 persons were concerned by these 160 incidents, 305 were susceptible to be contaminated, 54 to be irradiated and 10 presented the two susceptibilities. Measures confirmed the internal contamination for 112 persons. Among the 64 persons susceptible to be irradiated, 34 received a dose, and 4 a dose higher than $50 \mathrm{mSv}$. Due to the high

\footnotetext{
IRSN, LEDI, BP 17, 92262 Fontenay-aux-Roses Cedex. France.

IRSN, LAMR, BP 35. 78116 Le Vésinet Cedex, France.

IRSN, LSDOS, BP 35, 78116 Le Vésinet Cedex, France

DGSNR, 6 place du Colonel Bourgoin, 75572 Paris Cedex 12, France.

IRSN/DESTQ, BP 35, 78116 Le Vésinet Cedex, France.
} 
frequency of contamination incidents, it would be necessary to emphasize studies focused on radionuclides used in research laboratories and medical facilities in order to improve expertise in these sectors.

\section{Introduction}

Dans le domaine de la radioprotection de l'homme, l'expertise de l'IRSN est sollicitée lors de la survenue d'incidents susceptibles d'entraîner la contamination et/ou l'irradiation de travailleurs professionnellement exposés mais aussi de personnes du public. Afin d'améliorer cette expertise, il est essentiel de savoir quels sont les incidents susceptibles de survenir et quelles en sont les conséquences potentielles en termes de niveaux d'exposition. L'exploitation des données de l'ex-Office de protection contre les rayonnements ionisants (dont une partie a fusionné avec l'Institut de protection et de sûreté nucléaire en 2002 pour former l'Institut de radioprotection et de sûreté nucléaire) et de son prédécesseur le SCPRI (Service Central de Protection contre les Rayonnements Ionisants), présente dans ce domaine un intérêt particulier puisque pendant plus de 25 ans, les incidents de radioprotection susceptibles d'entraîner l'irradiation et/ou la contamination accidentelle d'une ou plusieurs personnes lui ont été signalés. Tous ces incidents ont fait l'objet de synthèses annuelles regroupant les différentes catégories d'incidents et présentées dans les rapports de l'OPRI. Cependant, aucune synthèse n'a encore été faite sur les conséquences en termes d'exposition pour les individus impliqués dans ces incidents. La présente étude se propose de faire ce bilan sur deux années, 1998 et 1999.

Seuls les incidents impliquant directement des individus et ayant donné lieu à des mesures d'activité, directes ou indirectes, et/ou à des estimations dosimétriques individuelles ont été retenus pour cette étude. Ce type d'incidents ne représente qu'une minorité de ceux signalés à l'OPRI dont la plupart ont concerné des anomalies de fonctionnement des installations (par exemple, des baisses de débit de ventilation) qui n'impliquaient pas directement des individus.

\section{Analyse des incidents}

\subsection{Description générale}

Au total, au cours des années 1998-1999, 160 incidents survenus en France et ayant fait l'objet de mesures et/ou d'estimations dosimétriques individuelles ont été signalés à l'OPRI. Ces 160 incidents ont concerné 369 individus. La majorité de ces incidents (91 soit $57 \%$ ) sont survenus dans l'industrie nucléaire dont 57 dans des centres nucléaires de production d'électricité. Les autres incidents se sont 


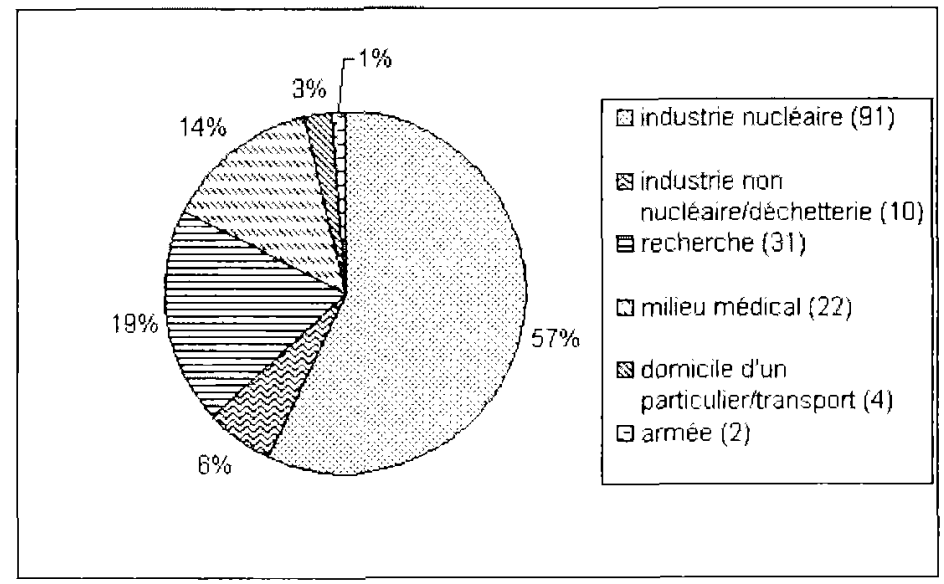

Figure 1 - Lieu de survenue des incidents.

Incidents location.

produits essentiellement dans des laboratoires de recherche (31 incidents, soit $19 \%$ ) et dans le secteur médical (22 incidents, soit $14 \%$ ). Parmi les 16 incidents restants, 8 sont survenus dans l'industrie non nucléaire, 2 dans une déchetterie ou une société de récupération, 1 au domicile d'un particulier, 3 dans les transports et 2 sur des sites appartenant à l'armée. La figure 1 illustre cette répartition. Ce large éventail de lieux de survenue reflète la diversité des secteurs concernés, notamment autres que l'industrie nucléaire.

Les incidents découverts lors de contrôles systématiques (portiques de sortie de zone contrôlée, surveillance dosimétrique externe, analyses radiotoxicologiques) ont été inclus dans l'étude même s'il n'a pas été possible de trouver l'origine de l'exposition. Les circonstances de découverte des incidents sont de deux types : lors d'un événement reconnu (par exemple le bris d'un flacon contenant une source non scellée dans un laboratoire de recherche) ou à la suite de résultats de mesure anormalement élevés.

Nous avons choisi d'exclure les incidents concernant un grand nombre d'individus dont le poids dans les statistiques auraient été trop importants. Ainsi, nous avons exclu l'affaire des "wagons de Valognes » survenue en 1998 et l'incendie du camion transportant des détecteurs de fumée contenant de l'américium survenu en 1999. De même, nous n'avons pas considéré huit incidents ayant donné lieu à des évacuations des bâtiments réacteurs des centrales nucléaires, deux survenus en 1998 et six en 1999. Nous avons également exclu trois incidents survenus hors de France en 1999. 


\subsection{Incidents survenus dans l'industrie nucléaire}

\subsubsection{Circonstances de découverte}

Au cours de la période d'étude, 91 incidents sont survenus dans ce secteur. Compte tenu de la fréquence des contrôles dans l'industrie nucléaire et de la sensibilité des appareils de détection de la radioactivité, la plupart de ces incidents (57 soit $63 \%$ ) ont été découverts lors de la recherche systématique de contamination individuelle ou environnementale, le plus souvent à l'aide des portiques situés en sortie de zone, ou lors du déclenchement d'une alarme de détection de la radioactivité ambiante. Lorsque l'incident donnait lieu à un événement reconnu (25 incidents soit $27 \%$ ), il s'agissait essentiellement de pertes de confinement avec dispersion d'une source ou d'un milieu contaminé. Pour neuf incidents, l'OPRI n'a pas eu connaissance précise des circonstances de leur découverte.

\subsubsection{Conséquences recherchées}

Les 91 incidents survenus dans l'industrie nucléaire ont concerné 187 travailleurs. Le tableau I décrit le type d'exposition suspectée, les investigations réalisées chez les travailleurs et leurs résultats. Parmi ces travailleurs, la grande majorité (177, soit $94,1 \%$ ) étaient susceptibles d'avoir été contaminés, $9(4,8 \%)$ d'avoir été irradiés et $1(0,6 \%)$ d'avoir été à la fois contaminé et irradié.

\subsubsection{Incidents de contamination}

Les 177 travailleurs susceptibles d'avoir été contaminés ont tous subi des examens pour évaluer leur niveau de contamination. Les examens ont été réalisés soit par mesure anthropogammamétrique soit par analyse radiotoxicologique des selles et/ou des urines. Au total, l'analyse radiotoxicologique des urines a été réalisée par l'exploitant pour 113 individus. L'exploitant a adressé à l'OPRI la moitié des urines de 104 individus pour réaliser une mesure d'intercomparaison. Par contre, seules 21 analyses des selles sur 69 réalisées par l'exploitant ont donné lieu à une intercomparaison. En ce qui concerne les anthropogammamétries, 4 travailleurs de l'industrie nucléaire sont venus à l'OPRI pour une intercomparaison. Au total, $63,8 \%$ des individus susceptibles d'être contaminés ont bénéficié d'une analyse des urines. Les incidents restants étaient dus à des radionucléides pour lesquels une analyse des selles était plus adaptée ou concernaient des contaminations externes sans qu'une contamination interne ait été mise en évidence lors des contrôles anthropogammamétriques.

Ainsi, les investigations réalisées ont confirmé l'existence d'une contamination interne chez 95 des 177 travailleurs concernés, soit 53,7\%. 


\section{TABLEAU I}

Incidents survenus dans l'industrie nucléaire. Incidents in the nuclear industry.

(a) Type d'exposition

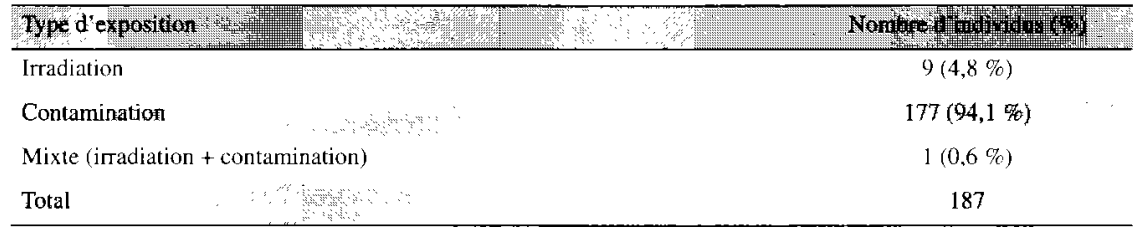

(b) Type d'investigations réalisées

\begin{tabular}{|c|c|}
\hline Irvestigations & 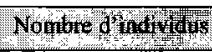 \\
\hline Dosimétrie film & 8 \\
\hline Dosimétrie opérationnelle & 7 \\
\hline Anthropogammamétrie OPRI & 4 \\
\hline Anthropoganmanêtrie exploitant & 142 \\
\hline Analyse radiotoxicologique des urines OPRI & 103 \\
\hline Analyse radiotoxicologique des urines exploitant & 112 \\
\hline Analyse radiotoxicologique des selles OPRI & 20 \\
\hline Analyse radiotoxicologique des selles explotant & 68 \\
\hline
\end{tabular}

(c) Résultats des investigations

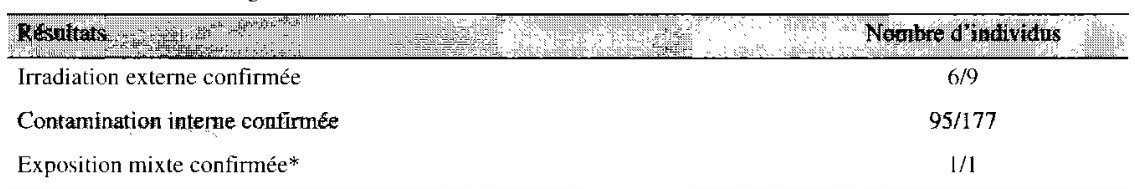

* Pour l’irradiation.

\subsubsection{Incidents d'irradiation}

Les quatre incidents susceptibles d'avoir entraîné des irradiations ont concerné neuf personnes. Toutes ont bénéficié d'une dosimétrie par film réglementaire, sauf pour un travailleur qui avait égaré son dosifilm. L'irradiation externe a été confirmée chez six travailleurs sur neuf ; la dose mesurée était faible, inférieure à $5 \mathrm{mSv}$, à l'exception d'un travailleur pour lequel une dose de $340 \mathrm{mSv}$ à l'organisme entier a été mesurée. Pour deux individus, dont celui qui avait égaré son film, le dosimètre opérationnel s'est avéré défaillant.

\subsubsection{Incidents susceptibles de donner lieu à des irradiations et à des contaminations}

Dans l'industrie nucléaire, un seul incident avait été initialement suspecté d'avoir provoqué à la fois une contamination interne et une irradiation. Il s'agissait d'une 
contamination vestimentaire. Les investigations n'ont cependant pas confirmé l'existence d'une contamination interne. La dose externe reçue par le travailleur au niveau de la malléole interne a été estimée à $131 \mathrm{mSv}$.

\subsection{Incidents survenus dans les laboratoires de recherche}

\subsubsection{Circonstances de découverte}

Parmi les 160 incidents signalés en 1998-1999, 31 se sont produits dans des laboratoires de recherche. Dans ce secteur, l'incident le plus fréquent est la dispersion d'une source non scellée, par exemple lors de la manipulation d'un flacon contenant une solution radioactive.

\subsubsection{Conséquences recherchées}

Les 31 incidents signalés dans des laboratoires de recherche ont impliqué 83 personnes. Le tableau II décrit le type d'exposition suspectée, les investigations réalisées chez les sujets concernés et leurs résultats. La plupart de ces incidents avaient pour conséquence possible une contamination interne des sujets. Globalement, $72 \%$ des sujets (61 sur 83) étaient susceptibles d'avoir été contaminés et $27 \%$ (22 sur 83) d'avoir été irradiés. Aucun cas d'exposition mixte n'était suspecté.

\subsubsection{Incidents de contamination}

La figure 2 montre la répartition des radionucléides impliqués dans les 28 incidents de contamination survenus dans les laboratoires de recherche. Les radionucléides les plus souvent en cause sont ceux qui font l'objet de la majorité des demandes de surveillance radiotoxicologique de routine dans ce domaine d'activité (Challetonde Vathaire et al., 1998). I1 s'agit du tritium, du carbone 14, du phosphore 32, du soufre 35 et de l'iode 125 . Les autres radionucléides ayant donné lieu à un incident étaient l'iode 131, le nickel 63, l'europium 152, le phosphore 33, l'uranium 234 et 238 et le plutonium 238 (un cas chacun). Deux incidents ont impliqué plusieurs radionucléides.

Les 61 individus susceptibles d'avoir été contaminés ont tous bénéficié d'une analyse radiotoxicologique urinaire réalisée à l'OPRI. Cet examen n'a confirmé la contamination que chez huit individus. Parmi ceux-ci, trois présentaient une contamination interne par de l'iode 125 , un par du soufre 35 , un par du carbone 14 et trois par de l'uranium. Un travailleur a bénéficié d'une analyse des selles qui a confirmé une contamination par du plutonium 238. 
TABLEAU II

Incidents survenus dans les laboratoires de recherche. Incidents in research laboratories.

(a) Type d'exposition

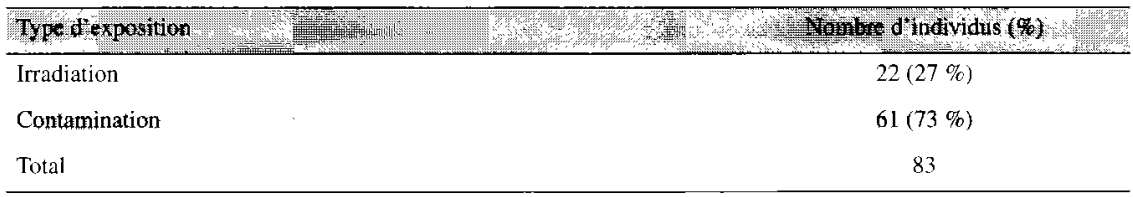

(b) Type d'investigations réalisées

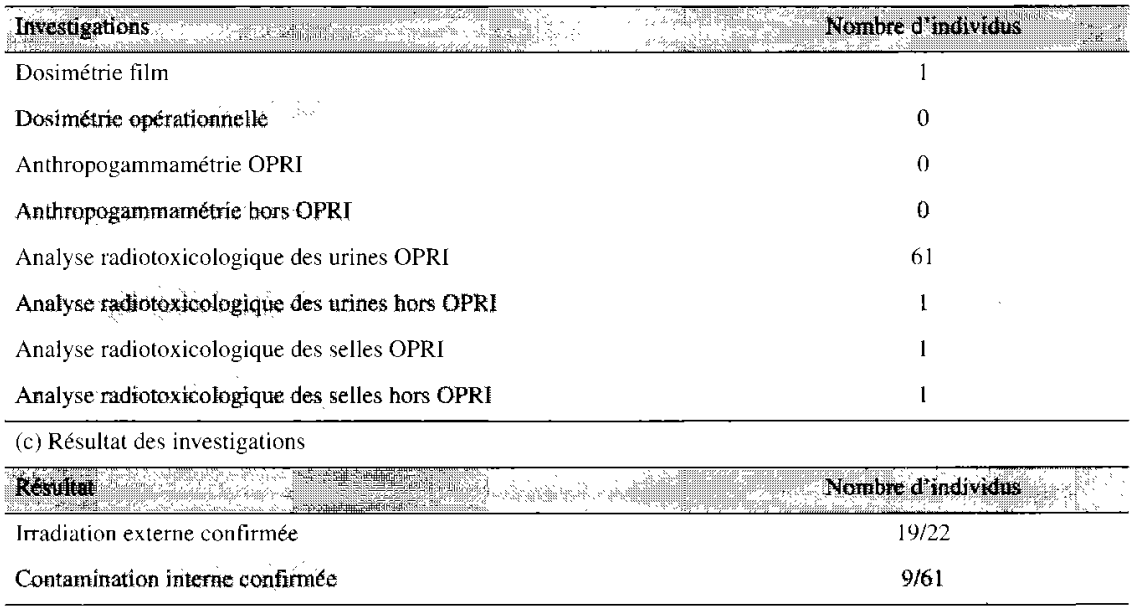

\subsubsection{Incidents d'irradiation}

Parmi les trois incidents susceptibles d'avoir provoqué des irradiations, deux sont survenus dans des laboratoires de physique et ont impliqué chacun un travailleur. Les sources en cause étaient d'une part une source scellée de césium 137, d'autre part des matériaux contaminés par du cobalt 60 . Le troisième incident est survenu dans un laboratoire de biologie et a impliqué 20 personnes, leur exposition résultait de la présence d'une source de radium « oubliée » dans un placard.

Sur les deux individus qui travaillaient dans des laboratoires de physique, l'un portait un dosifilm ayant enregistré une dose « corps entier» de $3 \mathrm{mSv}$. Les doses reçues localement au niveau des mains des deux travailleurs ont été estimées comprises entre 50 et $400 \mathrm{mSv}$.

Les vingt personnes susceptibles d'avoir été exposées à une source de radium ont bénéficié d'une reconstitution dosimétrique. La dose estimée a été considérée 


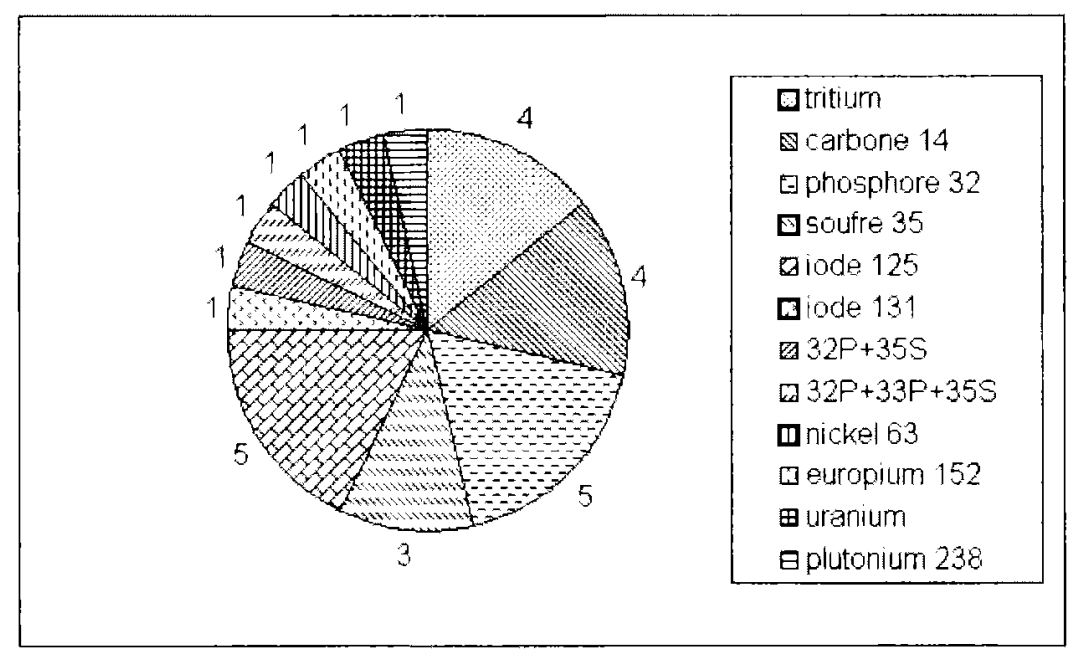

Figure 2-Nombre d'incidents de contamination en fonction du radionucléide impliqué dans les laboratoires de recherche.

Number of incidents of contamination in research laboratories function of the radionuclide concerned.

comme nulle pour trois d'entre elles, inférieure à $5 \mathrm{mSv}$ pour cinq, comprise entre à 5 et $20 \mathrm{mSv}$ pour huit, entre 20 et $50 \mathrm{mSv}$ pour cinq et supérieure à $50 \mathrm{mSv}$ pour deux d'entre elles.

\subsection{Incidents survenus dans le milieu médical}

Parmi les 22 incidents survenus dans le secteur médical, 9 ont eu pour cadre un service de médecine nucléaire, 7 des laboratoires d'analyse médicale, 2 un service de radiothérapie externe, 1 un service de curiethérapie et 1 un cabinet dentaire. Pour les 2 incidents restants, le type d'activité exercée n'était pas précisé.

\subsubsection{Circonstances de découverte}

Parmi les 22 incidents, 16 ont donné lieu à un événement reconnu. Dans 13 cas, il s'agissait de la perte de confinement d'une source non scellée, dans deux cas du dysfonctionnement d'un émetteur de rayonnements ionisants et dans un cas du dysfonctionnement d'un système de protection. Les six incidents restants ont été découverts à la suite d'une mesure de surveillance de routine, que ce soit par analyse radiotoxicologique des urines ( 4 incidents), sur un dosifilm réglementaire (un incident) ou lors d'un contrôle de contamination surfacique (un incident). 


\section{TABLEAU III}

Incidents survenus dans le secteur médical. Incidents in medical facilities.

(a) Type d'exposition

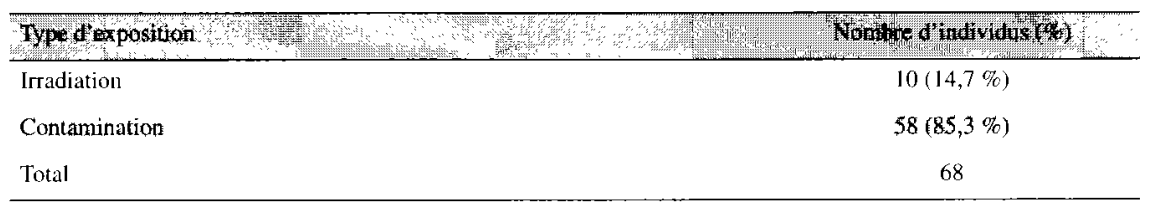

(b) Type d'investigations réalisées

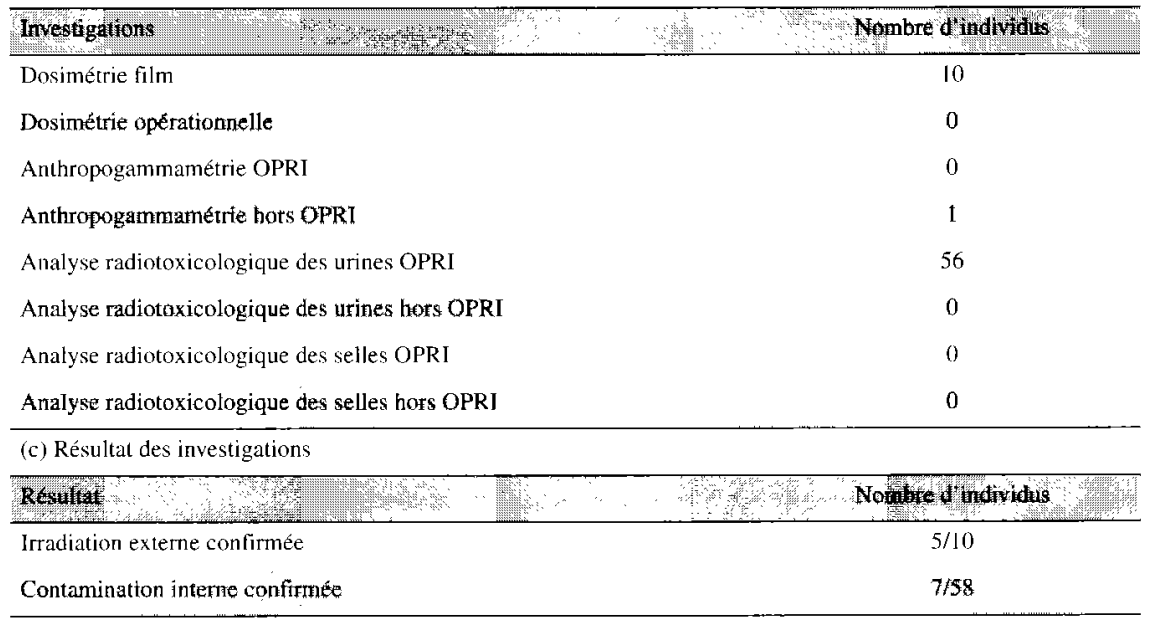

\subsubsection{Conséquences recherchées}

Les 22 incidents signalés dans le milieu médical ont impliqué 68 personnes. Le tableau III décrit le type d'exposition suspectée, les investigations réalisées chez les personnes concernées et leur résultats. Globalement, $85,3 \%$ des personnes (soit 58 sur 68 ) étaient susceptibles d'avoir été contaminées et 14,7\% (soit 10 sur 68) d'avoir été irradiées. Aucun cas d'exposition mixte n'était suspecté. Comme dans les laboratoires de recherche, la prépondérance du risque de contamination par rapport au risque d'irradiation s'explique par la perte de confinement lors de la manipulation d'une source radioactive non scellée.

\subsubsection{Incidents de contamination}

Dans le milieu médical, le radionucléide le plus souvent impliqué dans les incidents susceptibles de donner lieu à des contaminations est l'iode 131. Les 


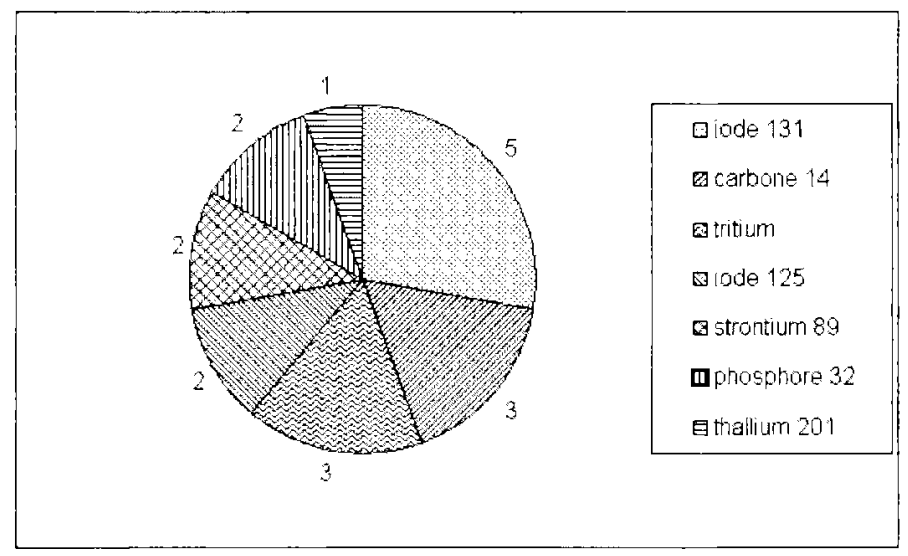

Figure 3 - Nombre d'incidents en fonction du radionucléide impliqué dans le milieu médical. Number of incidents in medical facilities function of the radionuclide concerned.

autres radionucléides sont les mêmes que ceux manipulés dans les laboratoires de recherche (tritium, carbone 14, iode 125, phosphore 32), auxquels s'ajoutent le strontium 89 et le thallium 201 utilisés dans les services de médecine nucléaire. La figure 3 illustre la distribution des 18 incidents de contamination en fonction du radionucléide impliqué.

Sur les 58 personnes susceptibles d'avoir été contaminées, 56 ont bénéficié d'une analyse radiotoxicologique des urines à l'OPRI (un flacon n'a pas été retourné) et une d'une anthropogammamétrie. Parmi ces 58 personnes, les mesures urinaires ont montré une contamination interne chez sept d'entre elles. Les radionucléides responsables de cette contamination étaient l'iode 131 (trois personnes), l'iode 125 (une personne), le carbone 14 (une personne), le thallium 201 (une personne) et le strontium 89 (une personne).

\subsubsection{Incidents d'irradiation}

En ce qui concerne les quatre incidents susceptibles de donner lieu à des irradiations, la source était dans deux cas un émetteur de rayons $X$, dans un cas du césium 137 utilisé en curiethérapie et dans un cas un générateur électrique de $18 \mathrm{MV}$.

Ces incidents ont impliqué dix travailleurs. Tous bénéficiaient d'une dosimétrie par film réglementaire. Les résultats ont confirmé l'irradiation chez cinq travailleurs. Deux travailleurs avaient reçu des doses inférieures à $1 \mathrm{mSv}$. Les doses reçues par les trois autres travailleurs étaient de 34,41 et $55 \mathrm{mSv}$. 


\subsection{Incidents survenus en dehors de l'industrie nucléaire, des laboratoires de recherche et du secteur médical}

Parmi les 16 incidents signalés en dehors de l'industrie nucléaire, des laboratoires de recherche et du secteur médical, huit sont advenus dans l'industrie non nucléaire, deux dans le secteur des déchets, un au domicile d'un particulier, trois dans les transports et deux sur des sites militaires.

\subsubsection{Circonstances de découverte}

Parmi les 16 incidents signalés, huit ont été découverts suite à de dysfonctionnements et quatre suite à des mesures. Pour les quatre incidents restants, l'OPRI n'a pas eu connaissance précise des circonstances de leur découverte.

\subsubsection{Conséquences recherchées}

Les 16 incidents signalés ont impliqué 31 personnes. Le tableau IV décrit le type d'exposition suspectée, les investigations réalisées chez les personnes concernées et leurs résultats. Parmi celles-ci, 9 étaient susceptibles d'avoir été irradiées, 13 d'avoir été contaminées et 9 d'avoir été irradiées et contaminées. Pour deux incidents, les renseignements concernant les investigations réalisées n'étaient pas disponibles.

\subsubsection{Incidents de contamination}

La nature des sources impliquées dans les huit incidents était très variée. Il s'agissait d'américium, d'uranium, de thorium, de carbone 14, d'iode 125 et 131. Pour deux incidents, la source n'était pas documentée. Parmi les treize personnes susceptibles d'être contaminées, sept ont bénéficié d'une analyse radiotoxicologique des urines à l'OPRI et deux d'une anthropogammamétrie. Les investigations réalisées ont confirmé la contamination pour une personne. Le radionucléide responsable de cette contamination était le carbone 14 .

\subsubsection{Incidents d'irradiation}

Les 6 incidents susceptibles de donner lieu à des irradiations sont tous survenus en milieu professionnel et ont concerné neuf travailleurs. Parmi ceux-ci, cinq ont bénéficié d'une dosimétrie réglementaire, complétée pour l'un d'entre eux par une dosimétrie opérationnelle. Les quatre autres travailleurs, qui n'étaient pas affectés à des travaux sous rayonnements ionisants, ont bénéficié d'une estimation dosimétrique à partir des éléments recueillis sur les circonstances de l'incident. 


\section{TABLEAU IV}

Incidents survenus en dehors de l'industrie nucléaire, des laboratoires de recherche et du secteur médical.

Incidents outside nuclear industry, research laboratories or medical facilities.

(a) Type d'exposition

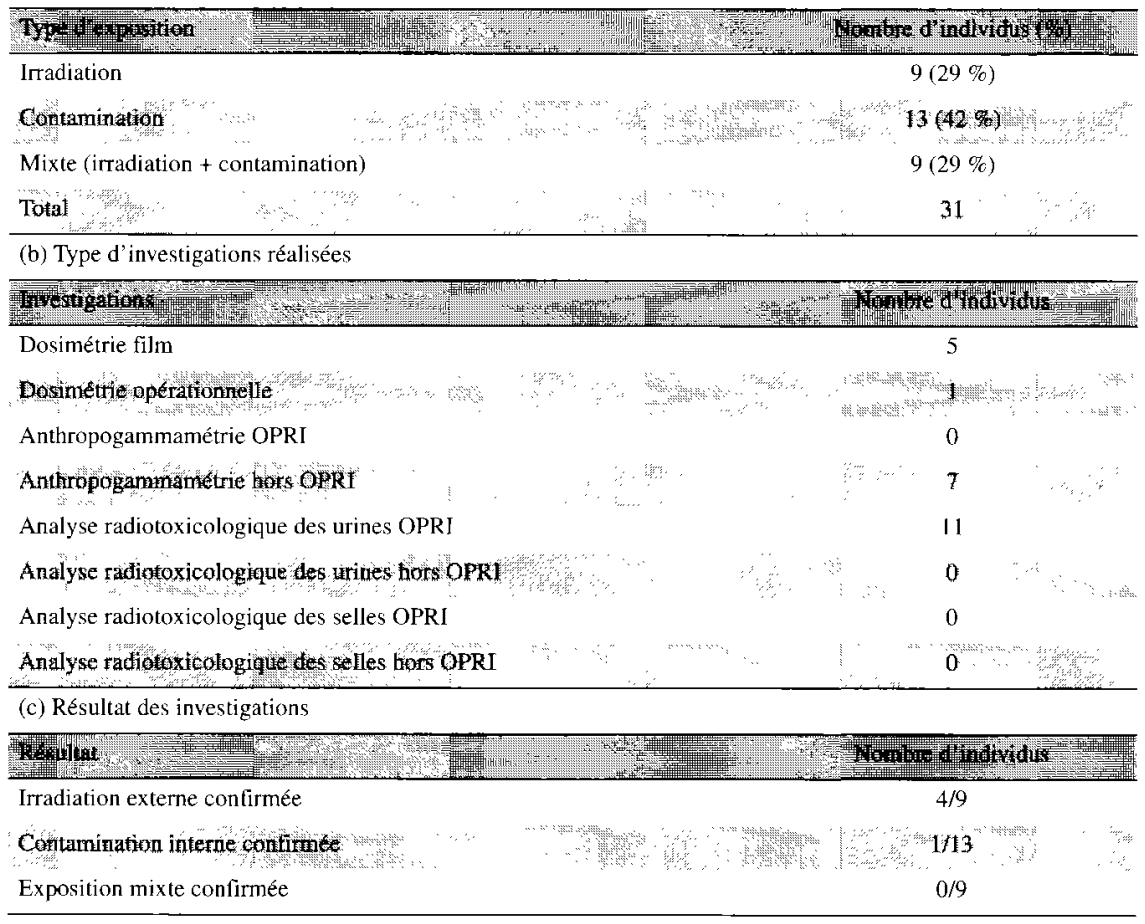

Ces mesures, ou ces estimations, ont conclu à une irradiation chez quatre travailleurs, inférieure à $2 \mathrm{mSv}$ chez trois d'entre eux. Pour un travailleur utilisant un appareil de gammagraphie, la dose était de $2,4 \mathrm{mSv}$ (dosimétrie film).

\subsubsection{Incidents susceptibles de donner lieu à des irradiations et à des contaminations}

Deux incidents susceptibles de donner lieu à une exposition mixte sont survenus, l'un au domicile d'un particulier et l'autre dans le secteur des transports. Dans les deux cas, il s'agissait de radium 226. Ces deux incidents ont concerné neuf personnes. Parmi celles-ci, cinq ont bénéficié d'une anthropogammamétrie et quatre d'analyses radiotoxicologiques urinaires à l'OPRI. Ces investigations n'ont pas confirmé les contaminations. 


\section{Discussion et conclusion}

La liste des incidents de radioprotection signalés à l'ex-OPRI pendant la période d'étude (1998-1999), ne saurait être exhaustive. Ils représentent cependant au niveau national l'expérience la plus large pendant la période d'étude puisqu'ils concernent l'ensemble des applications des rayonnements ionisants, que ce soit dans l'industrie nucléaire, l'industrie non nucléaire, les laboratoires ou le secteur médical.

Tous secteurs d'activité confondus, le nombre de personnes susceptibles d'être contaminées s'élevait à 305 , celui de celles susceptibles d'être irradiées à 54 et celui de celles susceptibles d'être à la fois contaminées et irradiées à 10 . Si l'on compare les deux années de la période d'étude, le nombre de personnes concernées était en augmentation, passant de 163 en 1998 à 206 en 1999.

En ce qui concerne le risque d'irradiation, près de la moitié des personnes concernées (35 sur 64) avaient effectivement été irradiées lors de l'incident. Les doses étaient loin d'être négligeables puisque pour 11 personnes la dose organisme entier mesurée ou estimée dépassait $20 \mathrm{mSv}$ voire $50 \mathrm{mSv}$ pour quatre d'entre elles. Trois personnes présentaient par ailleurs des irradiations localisées au niveau d'un membre entraînant des doses comprises entre 131 et $400 \mathrm{mSv}$. Le nombre élevé de personnes effectivement irradiées est dû à un incident où 17 personnes ont été irradiées par une source de radium méconnue.

Concernant le risque de contamination, le nombre de personnes susceptibles d'être contaminées, si l'on inclut celles présentant la double susceptibilité, était de 315. Les investigations réalisées ont confirmé l'existence d'une contamination interne dans $35,6 \%$ des cas (112/315). Le pourcentage de travailleurs présentant une contamination interne confirmée était élevé dans l'industrie nucléaire (53,7\% des cas) car les incidents étaient le plus souvent découverts, en l'absence d'événement notable, à la suite d'une mesure de contamination corporelle par des portiques situés en sortie de zone contrôlée.

La description dans cette étude des caractéristiques des incidents « tout venant " signalés en 1998 et 1999 apporte plusieurs informations. La première information concerne la dispersion des lieux de survenue des incidents. Si l'on considère le nombre de personnes concernées par ces incidents, la moitié l'a été en dehors de l'industrie nucléaire. En particulier, la fréquence des incidents survenant dans l'industrie non nucléaire avait déjà été signalée pour la série publiée en 1999 par l'unité de radiopathologie de l'Institut Curie (Cosset et al., 1999).

Cette étude montre par ailleurs que la grande majorité des incidents concerne des risques de contamination et non des risques d'irradiation. La gestion de ces 
incidents nécessite souvent une réponse très rapide, que ce soit pour la mise en route du recueil des excréta ou pour la réalisation d'examens anthropogammamétriques dans le cas des radionucléides à vie courte, mais également pour la prescription éventuelle d'un traitement spécifique ou non du contaminant. De plus, le nombre relativement important de personnes concernées dans les laboratoires de recherche et dans le secteur médical démontre la nécessité d'améliorer les connaissances sur les contaminations survenant dans ces secteurs. Le développement d'études spécifiques sur les radionucléides utilisés dans les laboratoires de recherche, mais aussi dans le domaine médical, est nécessaire pour permettre d'améliorer l'expertise en cas d'incident dans ces secteurs. En particulier, une meilleure connaissance des cinétiques de rétention et/ou d'excrétion de ces radionucléides doit permettre d'affiner les modèles permettant de calculer les activités incorporées à partir des mesures in vivo et/ou in vitro. Ces modèles pourraient être utilisés pour étendre la sélection des radionucléides traités dans la publication 78 de la CIPR (ICRP, 1998) à des isotopes responsables d'incidents en dehors de l'industrie nucléaire. Il faut cependant noter que les accidents professionnels entraînant des conséquences cliniques répertoriés dans le rapport 2000 de 1'UNSCEAR étaient des accidents d'irradiation (UNSCEAR, 2000).

\section{RÉFÉRENCES}

Challeton-de Vathaire C., Crescini D., Remenieras J., Biau A., Dubuquoy E., Cassagnou H., Bourguignon M., Masse R. (1998) Monitoring of workers occupationally exposed to radionuclides in France: results from February to August 1997 in the non-nuclear energy field, Radiat. Prot. Dosim. 79, 145-148.

Cosset J M, Perdereau B., Dubray B., Helfre S., Brixy F., Gongora R., Fourquer A. (1999) The 1951 1998 experience of the Paris Institut Curie Radiopathologie Unit : a preliminary report, $J$. Radiol. Prot. 19, 293-304.

ICRP (1998) Individual Monitoring for internal Exposure of Workers: Replacement of ICRP Publication 54, Ann. ICRP 27 (3-4).

Office de protection contre les rayonnements ionisants, Rapport d'activité 1995.

Office de protection contre les rayonnements ionisants, Rapport d'activité 1996.

Office de protection contre les rayonnements ionisants, Rapport d'activité 1997.

Office de protection contre les rayonnements ionisants, Rapport d'activité 1998.

Office de protection contre les rayonnements ionisants, Rapport d'activité 1999.

Office de protection contre les rayonnements ionisants, Rapport d'activité 2000.

UNSCEAR (2000) Sources and effects of ionizing radiation. UNSCEAR Report to the general assembly, New York. 